\title{
Close inspection of plasmon excitations in cuprate superconductors
}

\author{
Andrés Greco ${ }^{1,2}$, Hiroyuki Yamase ${ }^{3,4}$, and Matías Bejas ${ }^{1}$ \\ ${ }^{1}$ Facultad de Ciencias Exactas, Ingeniería y Agrimensura \\ and Instituto de Física Rosario (UNR-CONICET), \\ Av. Pellegrini 250, 2000 Rosario, Argentina \\ ${ }^{2}$ Max-Planck-Institut für Festkörperforschung, \\ Heisenbergstrasse 1, D-70569 Stuttgart, Germany \\ ${ }^{3}$ National Institute for Materials Science, Tsukuba 305-0047, Japan \\ ${ }^{4}$ Department of Condensed Matter Physics, Graduate School of Science, \\ Hokkaido University, Sapporo 060-0810, Japan
}

(Dated: July 8, 2020)

\begin{abstract}
Recently resonant inelastic x-ray scattering experiments reported fine details of the charge excitations around the in-plane momentum $\mathbf{q}_{\|}=(0,0)$ for various doping rates in electron-doped cuprates $\mathrm{La}_{2-\mathrm{x}} \mathrm{Ce}_{\mathrm{x}} \mathrm{CuO}_{4}$. We find that those new experimental data are well captured by acousticlike plasmon excitations in a microscopic study of the layered $t-J$ model with the long-range Coulomb interaction. The acousticlike plasmon is not a usual plasmon typical to the two-dimensional system, but has a small gap proportional to the interlayer hopping $t_{z}$.
\end{abstract}

PACS numbers: 


\section{INTRODUCTION}

The x-ray scattering technique is nowadays commonly used in cuprate high- $T_{c}$ superconductors. X-ray diffraction and resonant x-ray scattering revealed a charge-order tendency around the in-plane momentum $\mathbf{q}_{\|} \approx(0.6 \pi, 0)$ and $(0.5 \pi, 0)$ in hole-doped cuprates (hcuprates) $)^{1-3}$ and electron-doped cuprates (e-cuprates) $)^{4,5}$, respectively. Resonant inelastic x-ray scattering (RIXS) (Refs. 6-11) clarified a V-shaped dispersion of charge excitations around $\mathbf{q}_{\|}=(0,0)$, which extends up to a few eV.

While charge order was extensively discussed in terms of the so-called spin-charge stripes ${ }^{12}$ in cuprates ${ }^{13,14}$, those new x-ray experimental data were not in line with such a conventional scenario. The momentum of charge order and charge excitations are not correlated with that characterizing spin fluctuations, and in fact no spin order is accompanied by the charge order. Hence different types of charge orders were intensively explored for h-cuprates ${ }^{15-21}$, but the origin of the charge order is still under debate. For e-cuprates, a large- $N$ theory of the $t$ - $J$ model ${ }^{22}$ found that charge excitations are characterized by a dual structure in energy space. In a low-energy region, typically with a scale less than the exchange interaction $J$, various bond-charge excitations are present. Among others, bond-charge excitations with a $d$-wave symmetry exhibit a softening around $\mathbf{q}=(0.5 \pi, 0)$ (Refs. 22-24), consistent with the experiment data ${ }^{4,5}$. On the other hand, in the high-energy region, typically with a scale larger than $J$, plasmon excitations, i.e., collective on-site charge excitations, become dominant and explain the charge excitations observed around $\mathbf{q}_{\|}=(0,0)(\operatorname{Refs} .22,25,26)$.

In the context of the $t$ - $J$ model, both h- and e-cuprates are studied on an equal footing by changing the sign of the second nearest-neighbor hopping $t^{\prime}$ (Refs. 27,28). However, the extension of the large- $N$ theory of the $t-J$ model to h-cuprates cannot capture the observed charge-order tendency around $\mathbf{q}_{\|} \approx(0.6 \pi, 0)$ (Ref. 15). While the large- $N$ theory can be formulated in different schemes ${ }^{29-33}$, this drawback may not come from a large- $N$ scheme employed in Ref. 15. Rather it may lie in the fact that the charge-order tendency was observed inside the pseudogap state in h-cuprates, but the theoretical calculations ${ }^{22-24}$ were performed in a normal metallic state. Such calculations ${ }^{22-24}$ may be a reasonable description only for e-cuprates, where the pseudogap is absent or very weak ${ }^{34}$.

On the other hand, as in the case of e-cuprates, the large- $N$ theory captures the highenergy charge excitations observed in h-cuprates ${ }^{9}$ in terms of plasmons ${ }^{26}$. The plasmon 
excitations, therefore, seem universal in both h- and e-cuprates. However, the origin of the high-energy charge excitations are under debate and mainly three different scenarios are proposed: (i) a certain collective mode near a quantum phase transition, which is specific to e-cuprates ${ }^{7,35}$, (ii) intraband particle-hole excitations ${ }^{6,8,9}$ present in both e- and h-cuprates, and (iii) plasmon excitations with finite out-of-plane momentum $q_{z}^{10,22,25,26}$, which should be present in both e- and h-cuprates ${ }^{22,25,26}$.

Recently, $\mathrm{Cu} L_{3}$-edge RIXS experiments reported details of the high-energy charge excitations for e-cuprates $\mathrm{La}_{2-\mathrm{x}} \mathrm{Ce}_{\mathrm{x}} \mathrm{CuO}_{4}(\mathrm{LCCO})^{10,11}$. By using doping-concentration-gradient films, the authors in Ref. 11 reported fine details of the charge excitations as a function of doping, $q_{z}$, and $\mathbf{q}_{\|}$, which offer a stringent test of the plasmon scenario advocated in Refs. 10,25,26. This test is particularly important because some experiments cast doubt on the presence of the plasmons ${ }^{7-9,36,37}$. We find that those detailed data are well understood in terms of acousticlike plasmons obtained in the large- $N$ theory of the layered $t-J$ model.

\section{MODEL}

It is well known that cuprates are correlated electron systems and a minimal model of the $\mathrm{CuO}_{2}$ planes is the $t-J$ model ${ }^{38}$. To understand the high-energy charge excitations around $\mathbf{q}_{\|}=(0,0)$, the coupling between the adjacent planes is important as shown in a theoretical study $^{25}$, where a layered $t-J-V$ model was employed,

$$
\begin{aligned}
H & =-\sum_{i, j, \sigma} t_{i j} \tilde{c}_{i \sigma}^{\dagger} \tilde{c}_{j \sigma} \\
& +J \sum_{\langle i, j\rangle}\left(\vec{S}_{i} \cdot \vec{S}_{j}-\frac{1}{4} n_{i} n_{j}\right)+\frac{1}{2} \sum_{i, j} V_{i j} n_{i} n_{j}
\end{aligned}
$$

Here $\tilde{c}_{i \sigma}^{\dagger}$ and $\tilde{c}_{i \sigma}$ are the creation and annihilation operators, respectively, of electrons with spin $\sigma(=\uparrow, \downarrow)$ in the Fock space without any double occupancy, $n_{i}$ is the electron density operator, and $\vec{S}_{i}$ is the spin operator. The indices $i$ and $j$ run over the sites of a threedimensional lattice. The hopping $t_{i j}$ takes the value $t\left(t^{\prime}\right)$ between the first (second) nearestneighbor sites on a square lattice, and $t_{z}$ between the adjacent planes. $\langle i, j\rangle$ indicates a pair of nearest-neighbor sites on the square lattice and the exchange interaction $J$ is considered only inside the plane because the out-of-plane exchange term is much smaller than $J$ (Ref. 39). $V_{i j}$ is the Coulomb repulsion. 
Treating the nondouble occupancy constraint within a large- $N$ approximation ${ }^{25}$, the electronic quasiparticles disperse in momentum space as

$$
\varepsilon_{\mathbf{k}}=\varepsilon_{\mathbf{k}}^{\|}+\varepsilon_{\mathbf{k}}^{\perp}
$$

where the in-plane dispersion $\varepsilon_{\mathbf{k}}^{\|}$and the out-of-plane dispersion $\varepsilon_{\mathbf{k}}^{\perp}$ are given by, respectively,

$$
\begin{aligned}
\varepsilon_{\mathbf{k}}^{\|} & =-2\left(t \frac{\delta}{2}+\Delta\right)\left(\cos k_{x}+\cos k_{y}\right) \\
& -4 t^{\prime} \frac{\delta}{2} \cos k_{x} \cos k_{y}-\mu, \\
\varepsilon_{\mathbf{k}}^{\perp} & =2 t_{z} \frac{\delta}{2}\left(\cos k_{x}-\cos k_{y}\right)^{2} \cos k_{z} .
\end{aligned}
$$

The functional form $\left(\cos k_{x}-\cos k_{y}\right)^{2}$ in $\varepsilon_{\mathbf{k}}^{\perp}$ is frequently invoked for cuprates $^{40}$; see also Fig. 1 and Eqs. (4) and (7) in Ref. 41 for the interlayer hopping integrals in real space. Other forms for $\varepsilon_{\mathbf{k}}^{\perp}$, however, do not change the qualitative features of our results. Although the electronic dispersion looks like that in a free electron system, the hopping integrals $t, t^{\prime}$, and $t_{z}$ are renormalized by doping $\delta$ because of electron correlation effects. In addition, the term $\Delta$ in Eq. (3), which is proportional to $J$, is the mean-field value of the bond variables introduced to decouple the exchange term through a Hubbard-Stratonovich transformation. The value of $\Delta$ is computed self-consistently together with the chemical potential $\mu$ for a given $\delta$.

The term $V_{i j}$ in the Hamiltonian (1) describes the long-range Coulomb interaction, which satisfies Poisson's equation on a lattice. We solve it in momentum space and obtain ${ }^{42}$

$$
V(\mathbf{q})=\frac{V_{c}}{A\left(q_{x}, q_{y}\right)-\cos q_{z}},
$$

where $V_{c}=e^{2} d\left(2 \epsilon_{\perp} a^{2}\right)^{-1}$ and $A\left(q_{x}, q_{y}\right)=\alpha\left(2-\cos q_{x}-\cos q_{y}\right)+1$ with $\alpha=\frac{\tilde{\epsilon}}{(a / d)^{2}}$ and $\tilde{\epsilon}=\epsilon_{\|} / \epsilon_{\perp} ; \epsilon_{\|}$and $\epsilon_{\perp}$ are the dielectric constants parallel and perpendicular to the planes, respectively; $a$ and $d$ are the lattice constants in the planes and between the planes, respectively; and $e$ is the electric charge of electrons.

In the large- $N$ scheme, the density-density correlation function is renormalized already at leading order and can describe collective charge excitations; see Ref. 25 for a full formalism of the correlation function. We compute the imaginary part of the density-density correlation function as a function of $\mathbf{q}$ and $\omega$ for the parameters $t^{\prime} / t=0.30, t_{z} / t=0.03, \mathrm{~J} / t=0.3$, $V_{c} / t=8$, and $\alpha=3.2$. We consider 30 planes along the $z$ direction to get a reasonable 
resolution of the out-of-plane momentum transfer $q_{z}$; our $q_{z}$ is given by $q_{z}=2 \pi n_{z} / 30$ with $n_{z}$ being integer. Along the $x$ and $y$ direction we take the thermodynamic limit. The temperature is set zero.

Our results are compared with the data in Ref. 11, where momentum is given in units of $(2 \pi / a, 2 \pi / b, 2 \pi / c) ; a, b$, and $c$ are lattice constants. In the present theory, on the other hand, momentum is measured in units of $a^{-1}=b^{-1}=d^{-1}=1$. Since LCCO contains two planes in the unit cell, the distance between the adjacent planes is $d=c / 2$. For instance, the momentum $\mathbf{q}=(0.06,0,1.68)$ in Ref. 11 corresponds to $\mathbf{q}=(0.12,0,0.32) \pi$ in the first Brillouin zone in our theory. We set $t=750 \mathrm{meV}$ (Ref. 26) to describe energy in units of $\mathrm{eV}$.

In the present theory, the long-range Coulomb repulsion and the in-plane electronic correlations are treated on an equal footing. Hence our theoretical scheme is different from that in Ref. 10. In Ref. 10, the in-plane charge susceptibility was calculated first by determinant quantum Monte Carlo in the two-dimensional three-band Hubbard model and after that the effect of the long-range Coulomb interaction obtained in an layered electron gas model was incorporated into the dielectric function.

\section{RESULTS AND COMPARISON WITH EXPERIMENTS}

In Figs. 1(a)-1(c), we show the plasmon dispersion along $(\pi, \pi)-(0,0)-(\pi, 0)$ direction for $q_{z}=0,5 \pi / 15$ (close to an experimental value reported in Ref. 11), and $\pi$, respectively. The result for $q_{z}=0$ (upper panel) describes the optical plasmon mode, which is in good agreement with the plasmon frequency observed in cuprates ${ }^{43-45}$. Early experiments ${ }^{46}$ reported that the optical plasmon frequency increases with increasing doping, which is well reproduced in the $t-J$ model $^{25,47}$. For finite values of $q_{z}$ (middle and lower panels) the plasmon energy at $\mathbf{q}_{\|}=(0,0)$ suddenly drops. A close look at the results reveals the presence of a gap at $\mathbf{q}_{\|}=(0,0)$; see the insets in Figs. 1(b) and 1(c). Although the gapless excitations at $\mathbf{q}_{\|}=(0,0)$, namely acoustic plasmons were discussed for finite values of $q_{z}$ in Refs. 10 and 11 as well as early theoretical studies in a layered electron gas model ${ }^{48-50}$, the inter-layer hopping $t_{z}$ yields a finite gap at $\mathbf{q}_{\|}=(0,0)$ for a finite $q_{z}$ (Ref. 25). From Figs. 1(b) and 1(c), we predict a gap around $70 \mathrm{meV}$ for LCCO, i.e., much smaller than approximately $300 \mathrm{meV}$ reported for $\mathrm{Nd}_{2-\mathrm{x}} \mathrm{Ce}_{\mathrm{x}} \mathrm{CuO}_{4}(\mathrm{NCCO})^{7}$. This smaller gap originates mainly from 


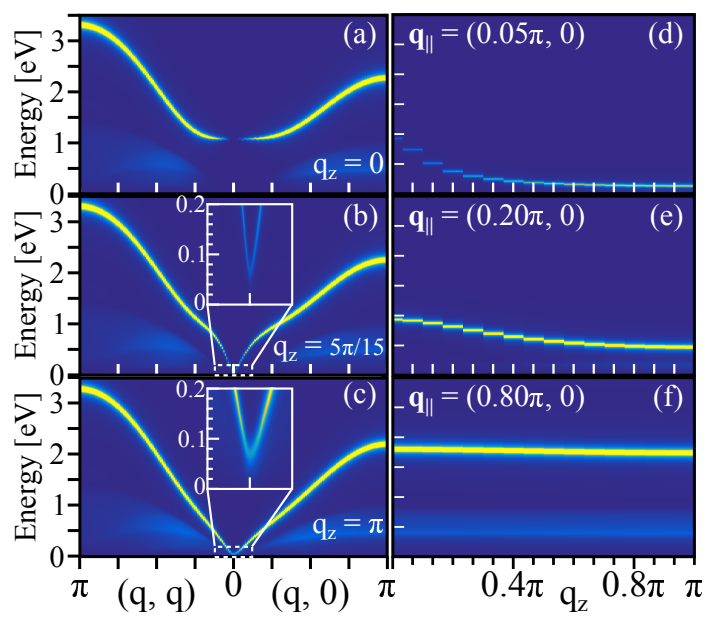

FIG. 1: Plasmon dispersion as a function of in-plane momentum along $(\pi, \pi)-(0,0)-(\pi, 0)$ direction for (a) $q_{z}=0$, (b) $5 \pi / 15$, and (c) $\pi$. The doping rate is $\delta=0.17$. The broadening is taken as $\Gamma=10^{-2} t$ to make the dispersion sharper. Faint spectral weight below $0.8 \mathrm{eV}$ corresponds to the particle-hole continuum and becomes broader with increasing $q$. The insets in (b) and (c) magnify a very low-energy region around $\mathbf{q}_{\|}=(0,0)$. Plasmon dispersion as a function of $q_{z}$ for $\mathbf{q}_{\|}=(0.05,0) \pi(\mathrm{d}),(0.2,0) \pi(\mathrm{e})$, and $(0.8,0) \pi(\mathrm{f})$.

the small value of $t_{z} / t=0.03$ in the present theory for LCCO and seems compatible with the experimental data ${ }^{10,11}$. Hence we consider that the experimental data are reasonably interpreted as acousticlike plasmons in the sense that they have a small gap at $\mathbf{q}_{\|}=(0,0)$.

Given that the crystal structures of LCCO and NCCO are the same, the reason why the value of $t_{z} / t$ in LCCO can become smaller than NCCO needs to be studied further, although new data for NCCO (Ref. 10) suggest a gap smaller than $300 \mathrm{meV}$; see the Supplemental Material in Ref. 10. It is also interesting to explore how the plasmon energy with finite $q_{z}$ changes by controlling the interlayer distance, e.g., by uniaxial pressure or intercalation of some elements between the layers, because the present theory predicts that the acousticlike plasmon energy is proportional to $t_{z}$ in a small $t_{z}$ region $^{25}$.

As clarified in Ref. 26, a characteristic feature of the plasmon excitations appears in its substantial $q_{z}$ dependence for a small in-plane momentum, which sharply distinguishes it from the usual intraband particle-hole excitations. Figures $1(\mathrm{~d})-1(\mathrm{f})$ show the plasmon dispersion as a function of $q_{z}$ for several choices of $\mathbf{q}_{\|}$. As seen in Figs. 1(a)-1(c), the plasmon intensity becomes very weak for a small $\mathbf{q}_{\|}$, but it is discernible in Fig. 1(d) that the plasmon energy and its intensity show a clear dependence on $q_{z}$. This $q_{z}$ dependence 


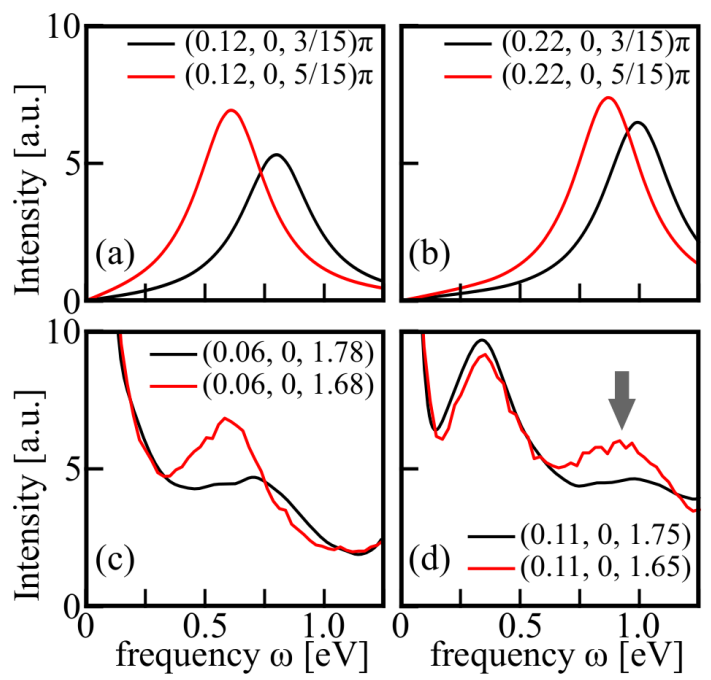

FIG. 2: Charge excitation spectra as a function of $\omega$ at $\mathbf{q}_{\|}=(0.12,0) \pi$ (a) and $(0.22,0) \pi$ (b) for $\delta=0.17$ and two different momenta $q_{z}$. (c) and (d) are the corresponding experimental data from Figs. 2(c) and 2(d) in Ref. 11. The arrow in (d) indicates excitations of charge origin. The peaks at $\omega \approx 0.3 \mathrm{eV}$ in $(\mathrm{d})$ correspond to paramagnon excitations and the peaks at $\omega=0$ in (c) and (d) are elastic; these features are out of a scope of the present work. While the in-plane momenta are set exactly the same as the experimental values, the values of $q_{z}$ are slightly different between our values and the experimental ones because we have a finite number of planes. For instance, $l=1.68$ in the experiment corresponds to $q_{z}=0.32 \pi$ which is close to $q_{z}=5 \pi / 15$ in the present calculations for 30 planes.

was reported in the experiment ${ }^{10}$. On the other hand, both the energy and the intensity become less sensitive to $q_{z}$ for larger values of $\mathbf{q}_{\|}$as shown in Figs. 1(e) and 1(f). Currently no experimental data is available for a larger value of $\mathbf{q}_{\|}$.

To make closer comparisons with the experimental data in Ref. 11, we show in Figs. 2(a) and (b) charge excitation spectra as a function of $\omega$ at $\mathbf{q}_{\|}=(0.12,0) \pi$ and $(0.22,0) \pi$ for $\delta=0.17$; experimental values of $q_{z}$ are finite and the charge excitation spectrum in $\mathbf{q}_{\|}-\omega$ space corresponds to Fig. 1(b), where the acousticlike plasmons are realized. A broadening $\Gamma=0.12 t$ is introduced phenomenologically in the calculations of the charge response. The peak energy at both momenta in Figs. 2(a) and 2(b) agrees very well with the experimental results Figs. 2(c) and 2(d), respectively. For a given value of $\mathbf{q}_{\|}$the experiment shows that the peak energy shifts upwards and, at the same time, the peak intensity decreases with decreasing $q_{z}$. As seen in Figs. 2(a) and 2(b) these two features are reproduced in 


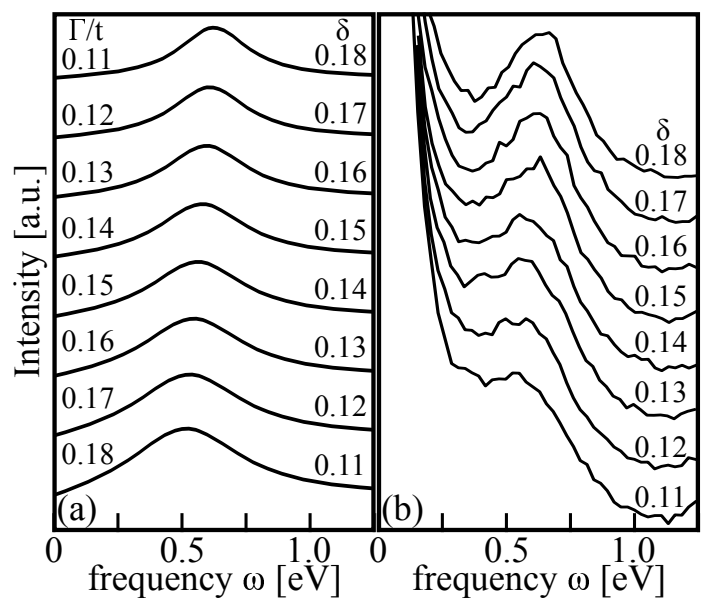

FIG. 3: (a) Charge excitation spectra as a function of $\omega$ at $\mathbf{q}=(0.12,0,5 / 15) \pi$ for different doping rates. The broadening $\Gamma$ is assumed to decrease with increasing doping. (b) The corresponding experimental results from Fig. 4(a) in Ref. 11. Although $q_{z}$ changes a little by changing doping in the experiment [see Fig. 4(g) in Ref. 11], such a change is very small and our value of $q_{z}=5 \pi / 15$ is appropriate for all doping.

the present theory. In addition, the difference of the peak energy between the two different values of $q_{z}$ decreases with increasing $\mathbf{q}_{\|}$[Figs. 2(c) and 2(d)], which is also captured in Figs. 2(a) and 2(b).

In Fig. 3(a) we show the doping dependence of the peak energy at $\mathbf{q}_{\|}=(0.12,0) \pi$. With increasing doping the peak energy increases monotonically in a way very similar to the experimental results [Fig. 3(b)]. In the experimental results of Fig. 3(b) the peak width decreases with increasing doping. This implies the suppression of incoherent features with doping. To reproduce this feature within the present theory, we have introduced a broadening $\Gamma$ (Ref. 26), which decreases with increasing doping. This $\Gamma$ mimics a broadening of the spectrum due to electron correlations obtained in a numerical study of the $t$ - $J$ model ${ }^{47}$.

Figure $4(\mathrm{a})$ shows results at $\mathbf{q}_{\|}=(0.12,0) \pi$ and $(0.08,0.08) \pi$ for doping rate $\delta=0.18$, where the peak position practically coincides at those two momenta. The peak energy agrees with the experimental data shown in Fig. 4(b) and the spectral shape of the peak would become essentially the same as the experimental data if the background is subtracted properly. In Fig. 4(c) we show the doping dependence of the peak energy for those two momenta (solid lines), which is practically linear in doping and reproduce the experimental results (see symbols). While a different doping dependence was discussed in Ref. 11 for a 

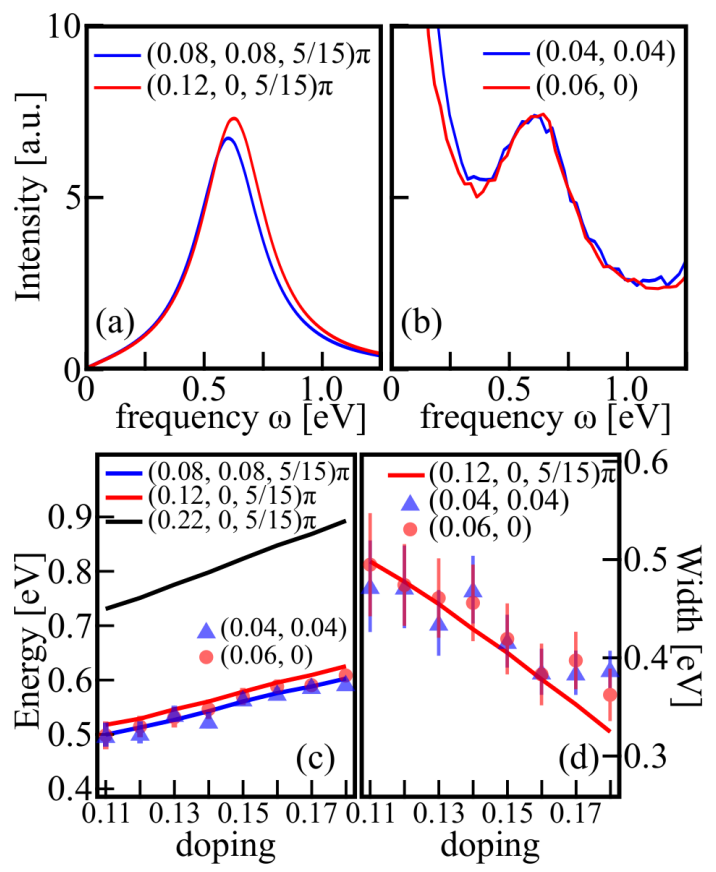

FIG. 4: (a) Excitation spectra at $\mathbf{q}=(0.12,0,5 / 15) \pi$ and $(0.08,0.08,5 / 15) \pi$ for $\delta=0.18$. (b) The corresponding experimental data from Fig. 4(d) in Ref. 11. (c) Doping dependence of the peak energy at $\mathbf{q}=(0.08,0.08,5 / 15) \pi$ (blue line),$(0.12,0,5 / 15) \pi$ (red line), and $(0.22,0,5 / 15) \pi$ (black line). (d) The peak width at $\mathbf{q}=(0.12,0,5 / 15) \pi$ as a function of doping. The symbols (triangles and circles) in (c) and (d) are the experimental data from Figs. 4(e) and 4(f) in Ref. 11, respectively.

large $\mathbf{q}_{\|}$such as $\mathbf{q}_{\|}=(0.22,0) \pi$, the present theory predicts essentially the same behavior also for $\mathbf{q}_{\|}=(0.22,0) \pi$ [black line in Fig. $4(\mathrm{c})$ ] at least in a doping region between 0.11 and 0.18 . In Fig. 4(d) we plot the full width at the half-maximum of the plasmon peak as a function of doping together with the experimental data, which confirms that our phenomenological $\Gamma$ actually works well.

\section{OUTLOOK}

We have shown that the acousticlike plasmons obtained in the layered $t$ - $J$ model explain well the high-energy charge excitation around $\mathbf{q}_{\|}=(0,0)$ for e-cuprates. For h-cuprates, however, it is not clear whether plasmons can be indeed present. The recent momentumresolved electron energy-loss spectroscopy ${ }^{36,37}$ cast doubt on the presence of plasmons in 
the h-cuprate $\mathrm{Bi}_{2.1} \mathrm{Sr}_{1.9} \mathrm{CaCu}_{2} \mathrm{O}_{8+\mathrm{x}}$. In those papers the authors claimed that the dynamical charge response is characterized by featureless and momentum-independent excitations. On the other hand, Ref. 9 reported dispersive high-energy charge excitations in h-cuprates $\mathrm{La}_{2-\mathrm{x}}(\mathrm{Br}, \mathrm{Sr})_{\mathrm{x}} \mathrm{CuO}_{4}$ by RIXS measurements. While the $q_{z}$ dependence, which is crucial to the plasmon scenario ${ }^{26}$, was not measured and the obtained dispersion was interpreted as incoherent charge excitations in Ref. 9, the observed dispersion as a function of in-plane momentum was explained in terms of the acousticlike plasmons ${ }^{26}$ similar to the present work. Our theory therefore implies that plasmons exist in both h- and e-cuprates in a rather symmetric way $^{26}$, although many properties such as the pseudogap, superconductivity, and antiferromagnetism exhibit a pronounced asymmetry ${ }^{34}$ between those systems. Further RIXS experiments in h-cuprates are important to clarify whether the high-energy charge excitations are indeed plasmons.

It is natural to ask a possible connection between plasmons and the pseudogap mainly observed in h-cuprates. In the present theory, the charge excitations exhibit a dual structure in energy space ${ }^{22}$, where plasmon excitations practically decouple from the low-energy excitations. Hence if low-energy charge fluctuations are related to the pseudogap in h-cuprates as frequently discussed in the literature ${ }^{51,52}$, we expect that in contrast to Refs. 36 and 37, highenergy charge excitations may not be the major source of the strange metallic properties. In fact, high-energy charge excitations extend to higher doping and have a doping dependence different from the pseudogap, superconductivity, and the strange metallic behavior.

Plasmons themselves are captured in a layered electron gas model ${ }^{48-50}$. In particular, one might obtain features similar to the present results. This does not imply that the electron gas model can be sufficient to understand plasmons in cuprates. In fact, as is well accepted, it is not an appropriate model of cuprates. Moreover, as reported in Refs. 1-5, there are also low-energy charge excitations, which seem different from plasmons. While the theoretical understanding of Refs. $1-3$ is still under debate ${ }^{15-21}$, we found that the present $t$ - $J$ model can capture very well the data presented in Refs. 4,5 in terms of $d$-wave bondcharge fluctuations ${ }^{2-24}$. This bond-charge physics cannot be captured in the electron gas model.

In addition, we point out two things which may distinguish the present theory from a standard weak coupling study to understand the plasmon physics in cuprates. i) The present theory predicts the vanishing of plasmons at half-filling whereas they remain even at half- 
filling in a weak coupling model. ii) When we apply the present theory to h-cuprates, we expect a doping dependence of the plasmon energy similar to Fig. 4(c). However, a weak coupling model would predict that the plasmon energy decreases with increasing doping.

\section{CONCLUSIONS}

Recalling that cuprate high- $T_{c}$ superconductivity is realized by charge carrier doping into the Mott insulator, the solid understanding of the charge dynamics is definitely indispensable to the cuprate physics. In particular, although plasmon excitations are obtained even in an electron gas model, it is important to clarify how well a realistic model of cuprates captures the experimental data. We have found that the acousticlike plasmons obtained in the layered $t-J$ model with the long-range Coulomb interaction explain even the fine details of charge excitations observed recently as a function of doping, in-plane and out-of-plane momenta for Ce-doped $\mathrm{La}_{2} \mathrm{CuO}_{4}$. The observed charge excitations around $\mathbf{q}_{\|}=(0,0)$ are likely due to acousticlike plasmons, although some experiments cast doubt on the presence of plasmons.

\section{Acknowledgments}

The authors thank M. Hepting, P. Horsch, B. Keimer, A. Nag, R. Zeyher, and K.-J. Zhou for very fruitful discussions. We thank P. Horsch for a critical reading of the manuscript. H. Y. acknowledges support by JSPS KAKENHI Grant No. JP15K05189 and JP18K18744. A. G. acknowledges the Japan Society for the Promotion of Science for a Short-term Invitational Fellowship program (S17027), under which this work was initiated, and Max-Planck Institute for Solid State Research in Stuttgart for hospitality and financial support.

1 G. Ghiringhelli, M. Le Tacon, M. Minola, S. Blanco-Canosa, C. Mazzoli, N. B. Brookes, G. M. De Luca, A. Frano, D. G. Hawthorn, F. He, et al., Science 337, 821 (2012).

2 J. Chang, E. Blackburn, A. T. Holmes, N. B. Christensen, J. Larsen, J. Mesot, R. Liang, D. A. Bonn, W. N. Hardy, A. Watenphul, et al., Nat. Phys. 8, 871 (2012).

3 A. J. Achkar, R. Sutarto, X. Mao, F. He, A. Frano, S. Blanco-Canosa, M. Le Tacon, G. Ghiringhelli, L. Braicovich, M. Minola, et al., Phys. Rev. Lett. 109, 167001 (2012). 
${ }^{4}$ E. H. da Silva Neto, R. Comin, F. He, R. Sutarto, Y. Jiang, R. L. Greene, G. A. Sawatzky, and A. Damascelli, Science 347, 282 (2015).

5 E. H. da Silva Neto, B. Yu, M. Minola, R. Sutarto, E. Schierle, F. Boschini, M. Zonno, M. Bluschke, J. Higgins, Y. Li, et al., Science Advances 2, e1600782 (2016).

${ }^{6}$ K. Ishii, K. Tsutsui, Y. Endoh, T. Tohyama, S. Maekawa, M. Hoesch, K. Kuzushita, M. Tsubota, T. Inami, J. Mizuki, et al., Phys. Rev. Lett. 94, 207003 (2005).

7 W. S. Lee, J. J. Lee, E. A. Nowadnick, S. Gerber, W. Tabis, S. W. Huang, V. N. Strocov, E. M. Motoyama, G. Yu, B. Moritz, et al., Nat. Phys. 10, 883 (2014).

8 K. Ishii, M. Fujita, T. Sasaki, M. Minola, G. Dellea, C. Mazzoli, K. Kummer, G. Ghiringhelli, L. Braicovich, T. Tohyama, et al., Nat. Commun. 5, 3714 (2014).

9 K. Ishii, T. Tohyama, S. Asano, K. Sato, M. Fujita, S. Wakimoto, K. Tustsui, S. Sota, J. Miyawaki, H. Niwa, et al., Phys. Rev. B 96, 115148 (2017).

10 M. Hepting, L. Chaix, E. W. Huang, R. Fumagalli, Y. Y. Peng, B. Moritz, K. Kummer, N. B. Brookes, W. C. Lee, M. Hashimoto, et al., Nature 563, 374 (2018).

11 J. Q. Lin, Jie Yuan, Kui Jin, Z. P. Yin, Gang Li, Ke-Jin Zhou, Xingye Lu, M. Dantz, Thorsten Schmitt, H. Ding, Haizhong Guo, M. P. M. Dean, and X. Liu, npj Quantum Materials 5, 4 (2020).

12 J. M. Tranquada, B. J. Sternlieb, J. D. Axe, Y. Nakamura, and S. Uchida, Nature (London) 375, 561 (1995).

13 S. A. Kivelson, I. P. Bindloss, E. Fradkin, V. Oganesyan, J. M. Tranquada, A. Kapitulnik, and C. Howald, Rev. Mod. Phys. 75, 1201 (2003).

14 M. Vojta, Adv. Phys. 58, 699 (2009).

15 M. Bejas, A. Greco, and H. Yamase, Phys. Rev. B 86, 224509 (2012).

16 A. Allais, J. Bauer, and S. Sachdev, Phys. Rev. B 90, 155114 (2014).

17 H. Meier, C. Pépin, M. Einenkel, and K. B. Efetov, Phys. Rev. B 89, 195115 (2014).

18 Y. Wang and A. Chubukov, Phys. Rev. B 90, 035149 (2014).

19 W. A. Atkinson, A. P. Kampf, and S. Bulut, New J. Phys. 17, 013025 (2015).

20 Y. Yamakawa and H. Kontani, Phys. Rev. Lett. 114, 257001 (2015).

21 V. Mishra and M. R. Norman, Phys. Rev. B 92, 060507(R) (2015).

22 M. Bejas, H. Yamase, and A. Greco, Phys. Rev. B 96, 214513 (2017).

23 H. Yamase, M. Bejas, and A. Greco, Europhys. Lett. 111, 57005 (2015). 
24 H. Yamase, M. Bejas, and A. Greco, Phys. Rev. B 99, 014513 (2019).

25 A. Greco, H. Yamase, and M. Bejas, Phys. Rev. B 94, 075139 (2016).

26 A. Greco, H. Yamase, and M. Bejas, Communications Physics 2, 3 (2019).

27 T. Tohyama and S. Maekawa, Phys. Rev. B 49, 3596 (1994).

28 R. J. Gooding, K. J. E. Vos, and P. W. Leung, Phys. Rev. B 50, 12866 (1994).

29 J. B. Marston and I. Affleck, Phys. Rev. B 39, 11538 (1989).

30 D. C. Morse and T. C. Lubensky, Phys. Rev. B 43, 10436 (1991).

31 Z. Wang, Int. J. Mod. Phys. B 6, 603 (1992).

32 R. Zeyher and M. L. Kulić, Phys. Rev. B 53, 2850 (1996).

33 M. Vojta and S. Sachdev, Phys. Rev. Lett. 83, 3916 (1999).

34 N. P. Armitage, P. Fournier, and R. L. Greene, Rev. Mod. Phys. 82, 2421 (2010).

35 G. Dellea, M. Minola, A. Galdi, D. Di Castro, C. Aruta, N. B. Brookes, C. J. Jia, C. Mazzoli, M. Moretti Sala, B. Moritz, et al., Phys. Rev. B 96, 115117 (2017).

36 M. Mitrano, A. A. Husain, S. Vig, A. Kogar, M. S. Rak, S. I. Rubeck, J. Schmalian, B. Uchoa, J. Schneeloch, R. Zhong, et al., Proceedings of the National Academy of Sciences 115, 5392 (2018).

37 A. A. Husain, M. Mitrano, M. S. Rak, S. Rubeck, B. Uchoa, K. March, C. Dwyer, J. Schneeloch, R. Zhong, G. D. Gu, et al., Phys. Rev. X 9, 041062 (2019).

38 F. C. Zhang and T. M. Rice, Phys. Rev. B 37, 3759 (1988).

39 T. Thio, T. R. Thurston, N. W. Preyer, P. J. Picone, M. A. Kastner, H. P. Jenssen, D. R. Gabbe, C. Y. Chen, R. J. Birgeneau, and A. Aharony, Phys. Rev. B 38, 905 (1988).

40 O. K. Andersen, A. I. Lichtenstein, O. Jepsen, and F. Paulsen, J. Phys. Chem. Solids 56, 1573 (1995)

41 H. Yamase and W. Metzner, Phys. Rev. B 73, 214517 (2006).

42 F. Becca, M. Tarquini, M. Grilli, and C. Di Castro, Phys. Rev. B 54, 12443 (1996).

43 E. J. Singley, D. N. Basov, K. Kurahashi, T. Uefuji, and K. Yamada, Phys. Rev. B 64, 224503 (2001).

44 N. Nücker, H. Romberg, S. Nakai, B. Scheerer, J. Fink, Y. F. Yan, and Z. X. Zhao, Phys. Rev. B 39, 12379 (1989).

45 H. Romberg, N. Nücker, J. Fink, T. Wolf, X. X. Xi, B. Koch, H. P. Geserich, M. Dürrler, W. Assmus, and B. Gegenheimer, Zeitschrift für Physik B Condensed Matter 78, 367 (1990). 
46 S. Uchida, T. Ido, H. Takagi, T. Arima, Y. Tokura, and S. Tajima, Phys. Rev. B 43, 7942 (1991).

47 P. Prelovšek and P. Horsch, Phys. Rev. B 60, R3735 (1999).

48 A. L. Fetter, Annals of Physics 88, 1 (1974).

49 V. Z. Kresin and H. Morawitz, Phys. Rev. B 37, 7854 (1988).

50 A. Bill, H. Morawitz, and V. Z. Kresin, Phys. Rev. B 68, 144519 (2003).

51 B. Keimer, S. A. Kivelson, M. R. Norman, S. Uchida, and J. Zaanen, Nature 518, 179 (2015).

52 B. Loret, N. Auvray, Y. Gallais, M. Cazayous, A. Forget, D. Colson, M.-H. Julien, I. Paul, M. Civelli, and A. Sacuto, Nat. Phys. 15, 771 (2019). 